\title{
MRI-Visual Order-Disorder Micellar Nanostructures for Smart Cancer Theranostics
}

\author{
Hirak Kumar Patra, Nisar Ul Khaliq, Thobias Romu, Emilia Wiechec, Magnus Borga, \\ Anthony P. F. Turner and Ashutosh Tiwari
}

\section{Linköping University Post Print}

\section{Tweet}

N.B.: When citing this work, cite the original article.

Original Publication:

Hirak Kumar Patra, Nisar Ul Khaliq, Thobias Romu, Emilia Wiechec, Magnus Borga, Anthony P. F. Turner and Ashutosh Tiwari, MRI-Visual Order-Disorder Micellar Nanostructures for Smart Cancer Theranostics, 2014, Advanced Healthcare Materials, (3), 4, 526-535.

http://dx.doi.org/10.1002/adhm.2013002255

Copyright (C) 1999-2014 John Wiley \& Sons, Inc. All Rights Reserved.

Publisher URL: http://onlinelibrary.wiley.com/

Postprint available at: Linköping University Electronic Press http://urn.kb.se/resolve?urn=urn:nbn:se:liu:diva-96875 


\section{MRI-visual order-disorder micellar nanostructures for smart cancer theranostics}

Hirak K Patra, ${ }^{1,2 \#}$, Nisar Ul Khaliq ${ }^{1 \#,}$ Thobias Romu ${ }^{3}$, Emilia Wiechec $^{2}$, Magnus Borga $^{3}$, Anthony PF Turner ${ }^{1}$ and Ashutosh Tiwari $^{{ }^{*}}$

${ }^{1}$ Biosensors and Bioelectronics Centre, Department of Physics, Chemistry and Biology (IFM), Linköping University, 58183 Linköping, Sweden

${ }^{2}$ Integrative Regenerative Medicine (IGEN) Center, Department of Clinical and Experimental Medicine (IKE), Linköping University, 58185 Linköping, Sweden

${ }^{3}$ Center for Medical Image Science and Visualization (CMIV), Department of Biomedical Engineering (IMT),

Linköping University, 58185 Linköping, Sweden

${ }^{\#}$ Authors contributed equally.

*Corresponding author.

E-mail: ashutosh.tiwari@liu.se, Tel: (+46)13-28-2395 and Fax: (+46) 13-13-7568.

Keywords: Custom-fit nanomedicine, Tumour microenvironment, Smart nanotheranostic; Personalised therapeutics. 


\section{Abstract}

Submitted to

This development of MRI-visual order-disorder structures for cancer nanomedicine explores pH-trigger mechanism for theragnosis of tumour hallmark functions. Superparamagnetic iron oxide nanoparticles (SPIONs) stabilised with amphiphilic poly(styrene)-b-poly(acrylic acid)-doxorubicin with folic acid (FA) surfacing is employed as a multi-functional approach to specifically target, diagnose and deliver drugs via a single nanoscopic platform for cancer therapy. The functional aspects of the micellar nanocomposite is investigated in vitro using human breast $\mathrm{SkBr} 3$ and colon cancer HCT116 cell lines for the delivery, release, localisation and anticancer activity of the drug. Our work shows, for the first time, concentration dependent $\mathrm{T}_{2}$-weighted MRI contrast for a monolayer of clustered cancer cells. The $\mathrm{pH}$ tunable order-disorder transition of the core-shell structure induces the relative changes in MRI contrast. The outcomes elucidate the potential of this material for smart cancer theranostics by delivering non-invasive real-time diagnosis, targeted therapy and monitoring the course and response of the action before, during and after the treatment regimen. 


\section{Introduction}

Submitted to

Considerable progress has already been made in the development of nanomaterials for drug delivery as well as disease diagnostics. One recent trend is the exploration of nanotheranostics, combining both diagnostic and therapeutic elements at the nano scale. ${ }^{[1,2]}$ Systems merging therapeutic agents, molecular targeting and diagnostic imaging capabilities are emerging as the next generation of multifunctional remedies. ${ }^{[3]}$ Drug targeting and delivery including the use of liposomes, micelles, dendrimers, nanotubes, and nanoparticles have been widely reported. ${ }^{[4-5]}$ However, research enabling real time tracking of such systems is in its infancy. ${ }^{[6]}$ The appropriate delivery of the drug to its target through site-specific stimuli such as inflammation, $\mathrm{pH}$ and ionic strength can further facilitate drug delivery from the carrier in a well-controlled manner. In addition, heterogeneous and adaptive diseases like cancer require fresh therapeutic protocols which are custom-fitted to the cancer type and individual patient. ${ }^{[7]}$

Current problems associated with conventional cancer chemotherapies include insolubility of drugs in aqueous medium, delivery of sub-therapeutic doses to target cells, lack of bioavailability and most importantly, non-specific toxicity to normal tissues. The recent contributions of nanotechnology address possible solutions to these conundrums. ${ }^{[8]}$ Likewise, challenges remain with respect to delivery to specific sites, real time tracking of the system and control over the release system after the drug has been transported to the target site. ${ }^{[9]}$ In the present contribution, we formulated a module using super paramagnetic iron oxide nanoparticles (SPION) as the core, stabilised with stimuli-responsive hydrazide-terminated poly(styrene)- $b$-poly(acrylic acid) block copolymer (PS-b-PAA) as the shell, which was loaded with the anticancer drug, doxorubicin (DOx) and with peripheral surfacing by folic acid. The covalent linkage of folate served to recognise the cancer site and the pH-sensitive DOx bonds delivered effective release of the drug at the tumour site. ${ }^{[10]}$

The SPION core of the module was chosen for its super paramagnetic properties, ${ }^{[11]}$ which facilitates non-invasive visualisation via magnetic resonance imaging. Flexible surface properties further enrich the current state of the art by core-shell nanoassembly. ${ }^{[12]}$ From a pharmacological perspective, we sought to endow the core with a reasonable circulation time, good lesion accumulation rate and high extravasation rate. ${ }^{[13]}$ The use of SPION as a core provides the potential for it to be guided to target sites by means of an external magnetic field and it can also be heated to provide local hyperthermia for cancer therapy. ${ }^{[14]}$ Moreover, the stimuli responsive order-disorder transition-enabled block copolymer, PS- $b$-PAA, had dual advantages from both the synthetic and application aspects. ${ }^{[15]}$ The stimuli-responsive order-disorder core-shell nanoassembly was used to solubilise the DOx in the hydrophobic core of the styrene and hence, reduce the possibility of unintended exposure and the consequent cytotoxicity for normal cells. 
Moreover, the fate of tumour progression and metastasis is heavily dependent on the associated microenvironment. ${ }^{[16]}$ The intrinsic acidic nature of the intratumour microenvironment is mainly guided by anaerobic and aerobic glycolysis followed by accumulation of lactic acid produced by the cancer cells. The $\mathrm{pH}$ dependent $\mathrm{T}_{2}$-weighted contrast properties of the synthesised SPION@PS-b-PAA-DOx/FA, in turn will sense the differential $\mathrm{pH}$ dependent cancer microenvironment and help in defining the case-by-case therapeutic protocol for personalised nanomedicine. ${ }^{[17]}$ In summary, we developed a $\mathrm{pH}$-triggered smart theranostic nanostructure for cancers which could be triggered by differential ionic properties of the tumour microenvironment. The methodology could greatly benefit the current investigation of new medications and diagnostics procedures for integrated cancer care. Furthermore, this methodology will help to improve the efficacy of the treatment by the early stage management of disease. This is very important in terms of point-of-care diagnosis, life-saving, timely treatment and cost cutting.

\section{Results and discussion}

\subsection{Synthesis and Characterisation of SPION@PS-b-PAA-DOx/FA}

The SPION@PS-b-PAA-DOx/FA was synthesised using a three-step procedure as shown in Scheme 1. In the first step, the hydrazide terminated PS- $b$-PAA was self-assembled around the SPION. The SPION contained $<1.0 \%$ short chains of PEG as stabilising/chelating agent, which in turn helped to form the core-shell assembly of SPION@PS-b-PAA. Further, in the second step, the DOx was conjugated with nanomicelles through the hydrazone linkage via the hydrazide moiety. The hydrazone linkage was formed by the carbonyl group $(>\mathrm{C}=\mathrm{O})$ of the DOx and the hydrazide $\left(\mathrm{R}^{-}\right)$terminal of the block copolymer. The carbonyl group is activated by proton $\mathrm{H}^{+}$and thereafter by the nucleophile addition of hydrazide formed intermediate that was converted to hydrazone linkage by the removal of a water molecule, SPION@PS- $b$-PAA-DOx. In the final step, folate was covalently attached with the carboxylic acid group of the poly(acrylic acid) block and amine group of vitamin B9 by amide linkage, SPION@PS- $b$-PAA-DOx/FA. The product was purified by dialysis against water to remove unreacted/excess of copolymer, DOx and folate. For the formation of the stable tuned nanomicelle, fresh medium was provided several times during dialysis prior to lyophilisation.

The respective samples were charactersed by ${ }^{1} \mathrm{H}$ NMR at $600 \mathrm{MHz}$ according to the specific chemical shift of PS- $b$-PAA and SPION@PS- $b$-PAA-DOx/FA (Figure 1). PS- $b$-PAA exhibited characteristic peaks in the ${ }^{1} \mathrm{H}$ NRM spectrum (Figure 1a). However, the peaks at $\delta 11.73$ and $\delta 10.62 \mathrm{ppm}$ represented the -COOH proton 
of the folic acid molecule in the SPION@PS- $b$-PAA-DOx/FA. The peaks at $\delta 7.88$ and $\delta 7.32$ ppm characterised the aromatic proton of the folic acid molecule. Whereas, the $\delta 6.87 \mathrm{ppm}$ proton shift was found at the terminal amine, and $\delta 5.87 \mathrm{ppm}$ represented aliphatics. The peaks at $\delta 3.71, \delta 1.53, \delta 1.25$ and $\delta 1.13 \mathrm{ppm}$ are indicative of the aliphatic protons in the SPION@PS-b-PAA-DOx/FA. The chemical shifts at $\delta 6.16 \mathrm{ppm}$ and $\delta 6.27 \mathrm{ppm}$ might show the aliphatic proton of the DOx molecule. The shift at $\delta 2.73 \mathrm{ppm}$ could be the $-\mathrm{OHCH}_{2}$ of DOx in the SPION@PS-b-PAA-DOx/FA. The peaks at $\delta 2.97 \mathrm{ppm}$ and $\delta 3.29 \mathrm{ppm}$ represented $-\mathrm{CH}_{2}$, and $-\mathrm{NH}_{2}$ of the DOx molecule. Likewise, the peak at $\delta 1.11 \mathrm{ppm}$ is ascribed to the proton of aliphatic $-\mathrm{CH}_{2}$ in the alicyclic ring of DOx molecule in the SPION@PS-b-PAA-DOx/FA (Figure 1b).

Earlier attempts to conjugate the DOx to a nanocarrier were successful in terms of chemistry, yet questionable with respect to the level of toxicity of the molecules used in the conjugation process. The folic acid functionalised nanomicelles were prepared using poly(benzyl L-aspartate)- $b$-poly(ethylene glycol) block copolymer and thereafter DOx was attached at the end for the tumour-targeted drug delivery. ${ }^{[18,19]}$ The attachment of terminal moieties, i.e., DOx and folic acid, was achieved by an extensive multi-step chemical procedure. Furthermore, hydrazone linkage with DOx was formed with anhydrous hydrazine, which is a highly toxic substance. ${ }^{[20]}$ In the current study, the design and synthesis are unique and novel because of the availability of terminal responsive functional groups on the amphiphilic SPION@PS-b-PAA core-shell composite for the attachment of DOx and vitamin B9. Folic acid is an essential water soluble vitamin (i.e., vitamin B9) mandatory for cell proliferation; however folate receptors are overexpressed on cancer cells. Due to the high binding capability of such vitamin, it provided a useful recognition molecule for tumour-targeted drug delivery. ${ }^{[21]}$ The folic acid binds to the folate receptors on the tumour cells by active targeting, which leads to receptor-mediated endocytosis and controlled release of drug delivered to the cancer cells. ${ }^{[22]}$ The hydrazone linkage is sensitive to low $\mathrm{pH}$ and hence the attached DOx in the nanocarrier is cleaved easily by the highly acidic environment in the tumour cells resulting in controlled release. Moreover, the inner hydrophobic shell of SPION@PS- $b$-PAA retains DOx without leakage during transportation. This mechanism helps to reduce the side effects of toxicity, for example, killing of normal cells and non-specific drug release. 


\subsection{Properties of SPION@PS-b-PAA-DOx/FA Nanomicelles}

Submitted to

The SPION@PS-b-PAA-DOx/FA composite formed core-shell micelles in aqueous solutions, balancing hydrophilic-to-hydrophobic functions. The hydrophobic property derived from the tendency of the polystyrene block of the copolymer to aggregate away from the aqueous phase to build the inner shell upon the SPION cores, whereas the hydrophilic part came from the water soluble blocks of PEG ligands and poly(acrylic acid) immersed in the water to formulate the outer shell of composite core-shell micelles. ${ }^{[18]}$ The micellar properties of SPION@PS-b-PAA-DOx/FA in phosphate buffer solution (PBS, pH 7.4) were determined by the critical aggregation concentration (CAC) using fluorescence spectroscopy with pyrene as a hydrophobic probe. A fixed shift (from 330 to $334 \mathrm{~nm}$ ) was observed in the excitation spectrum as a function of SPION@PS-b-PAADOx/FA concentration and this confirmed the self-assembly of SPION@PS- $b$-PAA-DOx/FA. It is established that styrene is selectively attached with the hydrophobic segments of the core-shell micelles structure in aqueous medium. ${ }^{[19,23]}$ Figure 2 shows the relation between intensity $I_{330}$ and $I_{334}$ ratio of the pyrene excitation spectra vs. logarithm of SPION@PS-b-PAA-DOx/FA concentration. The CAC of SPION@PS-b-PAA-DOx/FA core-shell micelles was dtermined to be $\sim 0.54 \mathrm{mg} / \mathrm{L}$ in PBS solution by the intersection of baseline and the tangent of the rapidly rising $I_{330} / I_{334}$ curve in Figure 2. It is well established that the micelles with lower CAC are highly stable in an aqueous environment, e.g., very high dissociation constant or long half life during prolonged circulation at extreme dilution during intravenous administration in the bloodstream. Hence, SPION@PS-b-PAA-DOx/FA core-shell micelles are very suitable as drug delivery carriers.

Dynamic Light Scattering (DLS) determined the size of the SPION@PS-B-PAA-DOX/FA core-shell micelles along with Transmission Electron Microscopy (TEM) as illustrated in right hand inset of Figure 2. The SPION@PS-b-PAA-DOx/FA core-shell micelles exhibited a hydrodynamic size distribution with a diameter ranging from 55 to $100 \mathrm{~nm}$. The average hydrodynamic diameter of SPION@PS-b-PAA-DOx/FA micelles was measured to be $78 \mathrm{~nm}$ with a polydispersity index of 0.06 . The size and morphology of the SPION@PS- $b$-PAADOx/FA micelles were further characterised using TEM. From the TEM study, the self-assembled SPION@PS$b$-PAA-DOx/FA micelles were seen to be well dispersed as individual micelles with a regular spherical shape and size of around 45-60 nm in diameter, as shown in inset to the right in Figure 2. The observed diameter of the micelles varied between measurements, cf., TEM gave smaller values than DLS. The DLS results reflect hydrodynamic diameter of the micelles, however TEM offered information about the morphology and size of micelles in a dry state. Thus, hydration of hydrophilic shell resulted in an increase in the micelle size during DLS measurements, while TEM gave information about the dehydrated micelle size and shape. Moreover, the size of the SPION@PS-b-PAA-DOx/FA micelles, i.e., $<100 \mathrm{~nm}$ is ideal for hydrophobic drug delivery. It is 
evident that micelles with a diameter $<150 \mathrm{~nm}$ avoid physical clearance by filtration in the lungs and spleen. The SPION@PS- $b$-PAA-DOx/FA nanomicelle size within such a range can also avoid renal clearance. ${ }^{[24]}$ The size of nanomicelle within this range could be promising for injectable anti-cancer drug delivery to the target moieties by extravasation, superior permeation and receptor-mediated endocytosis.

\subsection{Drug Release}

The DOx content entrapped into the SPION@PS-b-PAA-DOx/FA micelles was found to be 19 wt $\%$. Figure 3a shows the release profiles of DOx from SPION@PS-b-PAA-DOx/FA micelles at three different physiological $\mathrm{pH}$ points mimicking malignancy, metastatic, direct metastatic stages and intravenous administration into the bloodstream or release medium, i.e., PBS of $\mathrm{pH} 5.3,6.2$ and 7.4 at $37^{\circ} \mathrm{C}$ as a function of time. The study suggested that DOx encapsulated with SPION@PS-b-PAA-DOx/FA micelles revealed an initial burst, i.e., about 0-37 wt $\%$ at $\mathrm{pH} 5.3,0-23 \mathrm{wt} \%$ at $\mathrm{pH} 6.2$, and $0-12 \mathrm{wt} \%$ at $\mathrm{pH} 7.4$ of the initial loading amount, followed by a sustained release, approximately 37-92 $\mathrm{wt} \%$ at $\mathrm{pH} 5.3,23-72 \mathrm{wt} \%$ at $\mathrm{pH} 6.2$, and $12-32 \mathrm{wt} \%$ at $\mathrm{pH} 7.4 \mathrm{wt} \%$ of the initial loading amount and a non-release phase (cf., after $92 \mathrm{wt} \%$ at $\mathrm{pH} \mathrm{5.3,} 72 \mathrm{wt} \%$ at $\mathrm{pH} 6.2$, and $34 \mathrm{wt} \%$ at pH 7.4 wt $\%$ of the initial loading amount). The initial burst release of DOx from the micelles SPION@PS- $b$ PAA-DOx/FA at $\mathrm{pH} 5.3,6.2$ and 7.4 was attributed to DOx molecules attached within the shell or at the edge of the core and shell of the micelle.

Equally, the initial burst release of DOx from the micelles was attributed to DOx molecules located within the shell or at the core and shell interface of the micelle. The drug release equilibrium was reached after $72 \mathrm{~h}$. At malignancy $\mathrm{pH}$ points, the \% release of DOx was higher than at bloodstream $\mathrm{pH}$, which due to the cleavage of hydrazone linkages from the SPION@PS-b-PAA-DOx/FA micelles. Quick release of drug occurred once the SPION@PS- $b$-PAA-DOx/FA micelles were engulfed by cancer cells fllowing recognition of FA through a folate receptor mediated endocytosis process. Furthermore, an initial burst in release may be attributed to balancing the equilibrium between the inside and outside release settings. The slow release of the drug from the SPION@PS-b-PAA-DOx/FA micelles could be attributed to the hydrophobic-hydrophobic interactions between the drug molecules and the hydrophobic polystyrene inner shell of the micelles. Certain molecules of DOx might be adsorbed by tubing or solubilised in the polystyrene inner shell of the micelles, which resulted a non-release phase of drug release. ${ }^{[25]}$

The $\mathrm{pH}$ dependent magnetisation of SPION@PS-b-PAA-DOx/FA micelles was recorded at pH 5.3, 6.2 and 7.4. The variation in $\mathrm{M}-\mathrm{H}$ curves with respect to $\mathrm{pH}$ is shown in Figure 3b. It was observed that magnetisation of micelles at each $\mathrm{pH}$ point as chosen for the drug delivery study was virtually saturated at high 
magnetic field similar to crystallised SPIONs. ${ }^{[26]}$ At physiological temperatures, a weak magnetic hysteresis was observed at $\mathrm{pH} 7.4$, whereas, hysteresis loops are symmetric around the origin at both malignancy $\mathrm{pH}$ points. The effect of $\mathrm{pH}$ on the magnetisation is clearly perceptible in the evolution of the hysteresis loops, indicating that the SPION core in the SPION@PS-b-PAA-DOx/FA micelle introduced a superparamagnetic contribution by influencing the hydrodynamic volume of the $\mathrm{pH}$-responsive poly(acrylic acid) blocks. From the statistical mechanics, the magnetic particles in the liquid follow the relation-

$$
n=C \times e^{-p H / k T}
$$

Here,

$$
p=\frac{1}{4 \pi} \times \frac{\pi D^{3}}{6} \times M_{d}
$$

where $\mathrm{n}$ is particles per $\mathrm{cm}^{3}, \mathrm{C}$ is a constant, $p$ is the magnetic moment per particle, $\mathrm{H}$ is the magnetic field, $k$ is Boltzman constant, $\mathrm{T}$ is absolute temperature, $\mathrm{D}$ is diameter of particle and $\mathrm{M}_{\mathrm{d}}$ is magnetisation. From Equation (2), it is clear that superpramagnetic SPION@PS-b-PAA-DOx/FA micelles at pH 5.3, 6.2 and 7.4 are dependent on the size and density of the core-shell structure. This may be attributed to factors including variation of the oxygen stoichiometry of Fe-O-Fe from core to shell, variation in micellar size and changes in the magnetic moment. ${ }^{[27-29]}$ Thus, modules are released the DOx by three principal mechanisms, i.e., erosion, swelling and diffusion controlled by order-disorder core-shell structure. ${ }^{[30,31]}$

The SPION@PS-b-PAA-DOx/FA nanomicelle showed an initial burst release followed by a controlled drug delivery that obeys a diffusion-controlled mechanism; however, the diffusion rates at each stage of the DOx release varied greatly, suggesting two different processes may be taking place. Initially, drug release occurred relatively fast, up to $8 \mathrm{~h}$. It is evident that the drug present on the surface of inner shell or at the interface between the core and shell of micelle may be exposed to the release medium thus enabling order-disorder SPION@PS- $b$ PAA-DOx/FA transition using the pH-responsive hydrodynamic rate of poly(acrylic acid) blocks in the coreshell assembly (Figure 3c). This hypothesis was supported by the results from the bloodstream mimic $\mathrm{pH}$ at 7.4. In this case, diffusion of DOx was dramatically slower than $\mathrm{pH}$ points of metastatic spread and direct metastatic stages. 


\subsection{Cell Viability Assay}

Submitted to

The phasecontrast microscopy showed significant variation in the cell morphology for SPION@PS- $b$-PAADOx/FA and respective controls. The change in cellular morphology observed with respect to time and concentration. Visual inspection of cells showed cell debris with the treatment at the highest dose of $10 \mu \mathrm{M}$ (DOx loading concentration), which suggested that use of the SPION@PS-b-PAA-DOx/FA delivered the anticancer activity of doxorubicin. The anticancer effect and efficacy of SPION@PS- $b$-PAA-DOx/FA was tested by the 3-(4,5-dimethylthiazol-2-yl)-2,5-diphenyltetrasolim bromide (MTT) assay (Figure 4a). The assay was based on the fact that only viable cells have the active mitochondrial metabolic capacity to convert MTT into an optically measurable formazan derivative product, whereas dead cells or apoptotic cells are unable to convert MTT. ${ }^{[32]}$ Cell viability was compared to the untreated, composite/FA, free DOx and SPION@PS- $b$-PAADOx/FA. The cell viability (\%) was calculated based on the absorbance of the derivative produced by the untreated samples set at 100\%. Both cell lines showed $<5 \%$ cell viable, i.e., $3.6 \% \mathrm{SkBr} 3$ and $3.3 \% \mathrm{HCT} 116$ after treatment with $72 \mathrm{~h}$ while compared with the equimolar concentration of DOx (Figure 4b). The dynamics of cell death showed sustained decrease in \% of cell viability from 96.4 and 87.1 at $12 \mathrm{~h}$ to 5.4 and 15.6 at $72 \mathrm{~h}$ in case of SKBR3 and HCT116 cells, respectively. The gradual decrease in \% of cell viability implies sustained delivery of the drug from SPION@PS- $b$-PAA-DOx/FA from 8 to 72 h for effective anticancer chemotherapy. This increased potency and efficacy of the SPION@PS-b-PAA-DOx/FA might be due to the greater uptake and retention of the drug inside the intracellular environment. The MTT results were again in agreement with the cellular morphology changes through phase-contrast microscopy.

\subsection{Drug Uptake and Localisation}

The uptake and delivery of the drug by SPION@PS-b-PAA-DOx/FA was confirmed by confocal microscopy. One of the major challenges to select anti-cancer drugs is specifying the target molecule and the target site. There are two layers of selection, firstly, the drug should be specified to cell types or tissue types. ${ }^{[33]}$ In the second layer the drug should find the specific target including DNA, protein, RNA, biomolecule etc. within the intracellular environment. For example, we have used DOx as anti-cancer model drug for a SPION@PS- $b$-PAADOx/FA theranostic module. This has been used since 1960 for a wide spectrum of cancers such as rhabdomyosarcoma, breast cancer, adult acute leukemia, endometrial cancer, stomach cancer, cervical cancer and non-Hodgkin's lymphoma, to name a few. ${ }^{[34]}$ DOx is a DNA targeting intercalating agent that should be dimensionally fitted during the interaction. ${ }^{[35]}$ In order to investigate the criteria to serve as an effective 


\section{ADVANCED \\ HEATHCAARE \\ Submitted to \\ 14A 1 (1)}

theranostic SPION@PS-b-PAA-DOx/FA module, we have visualised the intracellular localisation of the drug (Figure 5).

The co-localisation study shows that the released DOx from nanocarrier was functionally effective to bind with the nuclear matter, i.e., DNA (Figure 5) in the SKBR3 and HCT116. The release of the drug from the carrier was necessary for the present system as otherwise it will be sterically forbidden for uptake through the nuclear membrane as well as intercalation within the DNA strands. The propensity for drug inclusion within the nucleus was illustrated by the overlap coefficient for the nucleus image versus the drug image of 0.75 and 0.89 for SKBR3 and HCT116, respectively.

\subsection{In vitro Magnetic Resonance Imaging (MRI)}

The $\mathrm{T}_{2}$-weighted MRI contrast properties have been evaluated using different concentrations in the SPION@PS$b$-PAA-DOx/FA and compared with the control phosphate buffer saline (PBS). The concentration dependent internalised contrast property of SPION@PS-b-PAA-DOx/FA is shown in Figure 6a, where the SkBr3 monolayer cell internalized the module shows a negative contrast effect $\left(R_{2}\right)$ in $T_{2}$-weighted images as $T_{2}$ signal intensity decreased with respect to the iron concentration present within the Composite-Dox/FA (SPION@PS-bPAA-DOx/FA). The probing of $\mathrm{pH}-$ dependent MR imaging properties is shown in Figure $\mathbf{6} \mathbf{b}$ with PBS as normal physiological buffer control. The negatively enhanced contrast MR imaging property has been shown by the Composite-Dox/FA with decrease in $\mathrm{pH}$ with respect to SPION. This $\mathrm{pH}$ dependent order-disorder property of the composite led to changes in extent of magnetisation as described in Figure 3 (b and c), is reflected in the MR imaging contrast. Hence we can identify the intracellular endocytic vescicles with low $\mathrm{pH}$ and the tumour microenvironment with differential $\mathrm{pH}$. The transverse $\left(1 / \mathrm{T}_{2}\right)$ relaxation rate of internalised micellar structures within cancer cells per unit was reflected in terms of MRI contrast. The $T_{2}$-weighted imaging defines the degree of change in the transverse relaxivities during the order-disorder formation. The Solomon-Bloembergen-Morgan equations ${ }^{[36]}$ defines the relationship between variables contributing to inner-sphere relaxivity including the number of inner-sphere water molecules $(\mathrm{q})$, the rotational tumbling time $\left(\tau_{\mathrm{R}}\right)$ and the residence lifetime of the inner-sphere (Figure 3c) water molecules $\left(\tau_{\mathrm{m}}\right)$. Likewise, a higher q value (swelling state of composite micelle) leads to larger relaxivity values of contrast agent during MR experiments. Water is also related to the mass transfer limitations in the micellar composite. Higher swelling increases the access of the drug and SPION core and certainly increases the $\mathrm{T}_{2}$ contrast with lowering $\mathrm{pH}$ from 7.4 to 5.2 . Therefore, by visually monitoring the change of $\mathrm{T}_{2}$-weighted contrast MR imaging, the profile of changing $\mathrm{pH}$ with respect to cancer progression can be obtained. The $\mathrm{T}_{2}$ map has been shown in Figure 6c, where the contrast ability in real time is illustrated. The 
concentration dependent intracellular MR imaging contrast properties are shown in the upper panel using monolayer a cell cluster of $\mathrm{SkBr} 3$ (i) The $\mathrm{pH}$ dependent $\mathrm{T} 2$ map shows the visual contrast of composite with respect to SPION (ii). The relative MR imaging contrast properties of SPION was not changing significantly for visual differentiation with respect to $\mathrm{pH}$, whereas there is a change $\left(\sim 5\right.$ times in terms of $\left.\mathrm{R}_{2}\right)$ in case of composite. Further, the folic acid surfacing also helps in the unintentional agglomeration of micellar structures (via electrostatic repulsion force of analogous charges) to protect in the differential MRI behavior as observed by Tromodrof ${ }^{[37]}$.

\section{Conclusions}

SPION@PS- $b$-PAA-DOx/FA core-shell nanocomposite micelles were synthesised as a stimuli-responsive targeted nanotheranostic module for the controlled release of drug into a tumour site with real-time monitoring possibilities through MRI. The anti-cancer drug DOx was conjugated to the block copolymer through a $\mathrm{pH}$ sensitive hydrazone linkage. The higher efficacy of the drug compared to the free DOx implies that the conjugated drug has been internalised at a higher rate and with increased retention time within the intracellular environment. Based on the earlier studies, we have hypothesised that FA helped to target and internalise the module, while the low $\mathrm{pH}$ intracellular environment assisted in cleaving the hydrazone bond to release DOx to pass through the nuclear membrane for efficient DNA binding. The confocal microscopic study showed intensive DOx accumulation in the nucleus via the effectively released DOx from the SPION@PS- $b$-PAADOx/FA after the endocytic uptake. These findings illustrate the potential of these biocompatible smart theranostic micellar nanostructures as a nontoxic, tumour-target specific, tumour-microenvironment sensitive, pH-responsive drug delivery system with provision for early stage tumour sensing, tracking and therapy for cells over expressed with folate receptors.

\section{Experimental}

\subsection{Materials}

Polystyrene- $b$-poly(acrylic acid) (PS- $b$-PA, $M_{\mathrm{n}}$ 9,800: $M_{\mathrm{n}}$ polystyrene $=6700$ and $M_{\mathrm{n}}$ poly $($ acrylic acid) $=3100)$, iron oxide $\left(\mathrm{Fe}_{3} \mathrm{O}_{4}, 10 \mathrm{~nm}, 45 \mathrm{emu} / \mathrm{g}\right.$ at $4.5 \mathrm{kOe}$, stabilised with polyethylene glycol, PEG ligand), doxorubicin hydrochloride (DOx), vitamin $\mathrm{B}_{9}$ (FA), $N$-hydroxysuccinimide (NHS), $N$-(3-dimethylaminopropyl)- $N^{\prime}-$ ethylcarbodiimide hydrochloride (EDC) and other supplementary reagents were obtained from Sigma-Aldrich, USA. Dialysis cassette (molecular weight cut-off, MWCO 8-10 kDa) was purchased from Thermo Scientific, 
U.S.A. SKBR3 and HCT116 cell lines were purchased from the American Type Culture Collection (ATCC, USA). All aqueous solutions were made in Milli-Q water (18.2 M $\Omega . \mathrm{cm})$.

\subsection{Synthesis of SPION@PS-b-PAA}

Hydrazide terminated PS- $b$-PA was prepared by the reduction method. $0.5 \mathrm{mM}$ of hydrazide terminated PS- $b$-PA was dissolved in $2 \mathrm{~mL}$ tetrahydrofuran (THF). Thereafter, $50 \mu \mathrm{L}$ of $\left(\mathrm{Fe}_{3} \mathrm{O}_{4}\right) \mathrm{aq}, 5 \mathrm{mg} / \mathrm{mL}$ was slowly added dropwise with stirring at room temperature. The assembly was kept in an inert atmosphere at room temperature for $48 \mathrm{~h}$. The solution was precipitated in a large excess of water. The collected SPION@PS- $b$-PAA was dissolved in water and dialysed in a dialysis tube against Milli-Q water. A soft SPION@PS-b-PAA was obtained after freeze-drying. SPION@PS- $b$-PAA yield $(\%)=98.25$.

\subsection{Synthesis of SPION@PS-b-PAA-DOx}

The pH-responsive bonding of DOx on SPION@PS- $b$-PAA was done using a hydrazone coupling reaction between the hydrazide terminus of SPION@PS- $b$-PAA and amine group of Dox. ${ }^{[34]}$ In a typical experiment, 50 mg of SPION@PS-b-PAA was reacted with DOx $(75 \mu \mathrm{L}, 10 \mathrm{mg} / \mathrm{mL})$ in aqueous medium. The reaction was carried out at $25{ }^{\circ} \mathrm{C}$ for $48 \mathrm{~h}$. The resulting SPION@PS- $b$-PAA-DOx was dialysed with a molecular weight cutoff $10 \mathrm{kDa}$ against phosphate buffer solution (PBS, $\mathrm{pH}$ 8.5). The drug loading was determined using UV-vis spectrophotometer at a wave length of $480 \mathrm{~nm}$.

\subsection{Preparation of SPION@PS-b-PAA-DOx/FA Nanomicelles}

The cancer cell-selective surface of SPION@PS-b-PAA-DOx was produced with vitamin $\mathrm{B}_{9}$ via coupling chemistry. ${ }^{[19]}$ Briefly, the acid group of SPION@PS- $b$-PAA was activated using EDC (0.38 mM) and NHS $(0.25 \mathrm{mM})$ for $45 \mathrm{~min}$ at room temperature with gentle stirring. The activated SPION@PS- $b$-PAA-DOx was then covalently linked to the folate $(0.125 \mathrm{mM})$ and incubated overnight under magnetic stirring at $25^{\circ} \mathrm{C}$. The resulting SPION@PS-b-PAA-DOx/FA micelles were prepared by the membrane dialysis with a molecular weight cut-off $10 \mathrm{kDa}$ against phosphate buffer solution (PBS, $\mathrm{pH} 8.5$ ). The DOx loading was further measured by UV-vis spectrophotometer at the wave length of $480 \mathrm{~nm}$. The conjugation of FA on SPION@PS- $b$-PAA-DOx was calculated by ${ }^{1}$ H NMR. SPION@PS- $b$-PAA yield $(\%)=98.25$; DOx loading =19\%; FA surfacing = 8 wt $\%$. 


\subsection{Characterisation}

Submitted to

The ${ }^{1} \mathrm{H}$ NMR spectrum of the samples was obtained with a $600 \mathrm{MHz}$ VARIAN NMR spectrometer using DMSO-d6 as solvent at $25{ }^{\circ} \mathrm{C}$. The absorbance measurements were observed using a Shimadzu 2450 UV-visible spectrophotometer. The calibration curve of absorbance was made at $480 \mathrm{~nm}$ against different DOx concentrations. The critical micelle concentration (CMC) of the SPION@PS-b-PAA-DOx/FA micelles was calculated using fluorescence spectra recorded on a fluorescence spectrometer (Photon Technology, Int.) with pyrene as a hydrophobic probe. The excitation spectra were recorded from 280 to $360 \mathrm{~nm}$ with an emission wavelength of $390 \mathrm{~nm} .{ }^{[21]}$ The TEM study was performed at $75 \mathrm{kV}$ for the characterisation of size and morphology of the nanomicelles. A drop of SPION@PS- $b$-PAA-DOx/FA micelles solution $(0.05 \mathrm{mg} / \mathrm{mL})$ was dropped on a 300 mesh copper grid coated with carbon and dried at room temperature prior to the observations taken using TEM (FEI Tecnai G2 Galadriel). The micellar size of SPION@PS- $b$-PAA-DOx/FA was determined by DLS using a Beckman Coulter PCS submicron particle size analyser at $90^{\circ}$. The concentration of the SPION@PS- $b$-PAA-DOx/FA solution was $0.25 \mathrm{mg} / \mathrm{mL}$. The magnetic study of the SPION@PS- $b$-PAADOx/FA nanomicelles was carried out with a vibrating sample magnetometer (VSM).

\subsection{Drug Release Study}

The drug release studies were performed in PBS at pH 5.3, 6.2 and 7.4 at $37^{\circ} \mathrm{C}$. Initially, $25 \mathrm{mg}$ of SPION@PS$b$-PAA-DOx/FA was dissolved in $5 \mathrm{~mL}$ respective PBS. Thereafter, the SPION@PS- $b$-PAA-DOx/FA micelles were transferred to a dialysis cassette with MWCO $10 \mathrm{kDa}$ and dialysed against PBS at three different physiological malignancy $\mathrm{pH}$ points from local, direct and metastatic spread stages release medium in a shaker with constant temperature $37^{\circ} \mathrm{C}$. Periodically, $2 \mathrm{~mL}$ of the released medium was taken and an equivalent volume of simultaneously maintainned fresh medium was added to restor the final volume. The analysis of released DOx was studied with a UV-vis spectrophotometer at $480 \mathrm{~nm}$.

\subsection{Cell Viability}

The cell viability upon SPION@PS- $b$-PAA/FA, DOx and SPION@PS-b-PAA-DOx/FA treatment was carried out by the MTT assay using human breast cancer (SKBR3) and human colon carcinoma (HCT116) cell lines. All cancer cell lines were cultured in RPMI-1640 medium supplemented with 10\% FCS. For the measurement of cell viability upon treatment with all respective materials, $1 \times 10^{4}$ cells were placed of 96-well-plates in each well and left overnight at $37{ }^{\circ} \mathrm{C}$. The cells were treated with various concentrations of DOx, composite-DOx/FA 
or composite/FA nanomicelles for $24 \mathrm{~h}$ or left untreated. After the treatment, MTT was added to the cells followed by incubation for $3 \mathrm{~h}$ at $37{ }^{\circ} \mathrm{C}$. The cells were centrifuged to the bottom of the plate and the cell culture medium was discarded. Next, $150 \mu \mathrm{L}$ of ethanol/DMSO in 1: 1 ratio per well was added for 30 min. The plate was measured by a spectrophotometer equipped with $\lambda=570 \mathrm{~nm}$ and $\lambda=630 \mathrm{~nm}$ filters. All the measurements were carried out in the triplicate and the data are shown as a mean of $\pm \mathrm{SD}$.

\subsection{Confocal Microscopy}

Visualisation of DOx uptake was performed using the LSM-700 confocal microscope (Carl Zeiss, Jena, Germany) equipped with appropriate filters. Briefly, the cells were seeded in a six well plate $\left(1 \times 10^{4}\right.$ cells/ well $)$ and grown overnight. The cells were treated with the drug loaded composite-DOx/FA for $24 \mathrm{~h}$ or left untreated. The cells were then washed with PBS and fixed with 4\% paraformaldehyde. The nuclei were stained using 4',6diamidino-2-phenylindole dihydrochloride (DAPI). The propensity of the drug inclusion within the nucleus was calculated by measuring the overlap co-efficient using Zen 2009, the image acquisition and analysis software.

\subsection{In vitro Magnetic Resonance Imaging (MRI)}

The in vitro MRI experiments were performed using a 1.5 Tesla Philips Achieva imaging system. The magnetic microscopic transverse relaxation time $\left(T_{2}\right)$ weighted images were measured for various concentrations $(10,25$, 50, 100, 250, and $250 \mu \mathrm{g} / \mathrm{ml})$. A pH dependent study has been done using $250 \mu \mathrm{g} / \mathrm{ml}$ of SPION@PS-b-PAADox/FA at pH 7.2, 6.2 and 5.3. All the samples were run under Turbo Gradient Spin Echo (GRASE) sequences at room temperature. The $T_{2}$ weighted intensities were measured in the region of interest. The $T_{2}$ weighted images were acquired using standard methods ${ }^{[38]}$ with small modifications. In brief, the parameters are as follows: Repetition time $(\mathrm{TR})=5000 \mathrm{~ms}$; echo time $(\mathrm{TE})=20$ to $200 \mathrm{~ms}$ with an interval of $20 \mathrm{~ms}$; Flip angle = $90^{\circ} ;$ Matrix $=200 \times 99($ Feet-Head $\times$ Right - Left $) ;$ FOV $=200 \times 100 \mathrm{~mm}$; slice thickness $=1.5 \mathrm{~mm}$; coil $=$ small flex coil; NSA (averages) $=3$.

The intracellular uptake and the concentration dependent distribution of SPION@PS-b-PAA-Dox/FA for the determination of MRI contrast was evaluated using human breast carcinoma, $\mathrm{SkBr} 3$ cell lines. $\mathrm{SkBr} 3$ cells were cultured in 96 well plate with RPMI-1640 with $10 \%$ FCS for 24 hours at $37^{\circ} \mathrm{C}$. The cells were treated for 3 hours with various concentrations with final Fe concentration of 10, 25, 50, 100 and $250 \mu \mathrm{g} / \mathrm{mL}$. The cells were then washed twice with PBS and fixed with 2\% paraformaldehyde in PBS followed by incubation for one hour at $4^{\circ} \mathrm{C}$. The cells were then washed twice and kept in PBS for MRI experiment as mentioned above. 
The relaxation rates for each sample were computed using Matlab R2012b (8.0.0.783, Mathworks, US) with references from the earlier reports. In brief, each image-stack collected within one acquisition by reading a series of echoes is considered for comparing the intensities. We have computed the relative difference between voxels ( $3 \mathrm{~d}$ pixels) in each sample by extracting the pixel index. The detailed script that has been used for the evaluation of the mean, median and standard deviations of the pixel intensities has been given in the supplementary section (S1).

\section{Acknowledgements}

The authors thank to Linköping University (LiU), Sweden for providing facilities. We sincerely thank to Center for Medical Image Science and Visualization (CMIV) at LiU for MRI facilities. We also wish to acknowledge the IGEN (Post-Doctoral Fellowship), European Commission (PIIF-GA-2009-254955) \& LIST, Linköping University for generous financial support to carry out this research.

Received: ((will be filled in by the editorial staff))

Revised: ((will be filled in by the editorial staff)) Published online: ((will be filled in by the editorial staff)) 


\section{References}

Submitted to

[1] A. Tiwari, A. Tiwari (Eds.), in Nanomaterials in Drug Delivery, Imaging and Tissue Engineering, WileyScrivener, USA 2013

[2] Kievit FM, Zhang M. Adv Mater 2011, 23, 217.

[3] D.E. Lee, H. Koo, I.C. Sun, J.H. Ryu, K. Kim, I.C. Kwon, Chem. Soc. Rev. 2012, 41, 2656.

[4] M. Beija, R. Salvayre, L.N. Viguerie, J.D. Marty, Trends Biotechnol 2012, 30, 485.

[5] H.K. Patra, A.K. Dasgupta, S. Sarkar, I. Biswas, A. Chattopadhyay, Cancer Nanotechnology 2011, 2, 37.

[6] Xie J, Chen K, Huang J, Lee S, Wang J, Gao J et al. Biomaterials 2010, 31, 3016.

[7] B. Sumer, J. Gao, Nanomedicine 2008, 3, 137.

[8] E.L. Romero, M.J. Morilla, Adv. Drug Deliv. Rev. 2010, 62, 576.

[9] K. Malinowsky, C. Wolff, S. Gündisch, D. Berg, K.F. Becker, J. Cancer 2011, 2, 26.

[10] J.Z. Du, X.J. Du, C.Q. Mao, J. Wang, J. Am. Chem. Soc. 2011, 133, 17560.

[11] S. Sulek, B. Mammadov, D.I. Mahcicek, H. Sozeri, E. Atalar, A.B. Tekinay, J. Mater. Chem. 2011, 21, 15157.

[12] S. Laurent, D. Forge, M. Port, A. Roch, C. Robic, L.E. Vander, Chem. Rev. 2008, 108, 2064.

[13] A.C. Silva, T.R. Oliveira, J.B. Mamani, S.M. Malheiros, L. Malavolta, L.F. Pavon, Int. J. Nanomed. 2011, $6,591$.

[14] J. Xie, K. Chen, J. Huang, S. Lee, J. Wang, J. Gao, Biomaterials 2010, 31, 3016.

[15] Q. Zhang, N.R. Ko, J.K. Oh, Chem Commun 2012, 48, 7542.

[16] G. Mikhaylov, U. Mikac, A.A. Magaeva, O. Vasiljeva, Nature nanotechnology 2011, 6, 594.

[17] T. Lammers, L.Y. Rizzo, G. Storm, F. Kiessling, Clinical Cancer Research 2012, $18,4889$.

[18] D. Chen, Z. Luo, N. Li, J.Y. Lee, J. Xie, and J. Lu, Advanced Functional Materials 2013, doi: 10.1002/adfm.201300411.

[19] M. Prabaharan, J.J. Grailer, S. Pilla, D.A. Steeber, S. Gong, Biomaterials 2009, 30, 3009.

[20] S. M. Hussain, J.M. Frazier. Toxicol Sci 2002, 69, 424

[21] G.H. Li, P.P. Yang, Z.S. Gao, Y.Q. Zhu. Colloid Polym Sci. 2012, 290, 1825.

[22] M. Prabaharan, J.J. Grailer, S. Pilla, D.A. Steeber, S. Gong, Macromol Biosci, Macromol Biosci 2012, 9 , 15.

[23] L. Zhang, A. Eisenberg, A, Science, 1995, 268, 23.

[24] J. Ai,Y. Xu, D. Li, Z. Liu, E. Wang. Talanta 2012, 101, 32.

[25] P.S. Low, S.A. Kularatne, Curr. Opin. Chem. Biol. 2009, 13, 256. 
[26] H.S. Choi, W. Liu, P. Misra, E. Tanaka, J.P. Zimmer, B.I. Ipe, Nat. Biotechnol. 2007, $25,1165$.

[27] L.W. Starmans, D. Burdinski, N.P. Haex, R.P. Moonen, G.J. Strijkers, K. Nicolay, H. Grüll, PloS one, 2013, 8, e57335.

[28] M. Tadić, V. Kusigerski, D. Marković, M. Panjan, I. Milošević, V. Spasojević, Journal of Alloys and Compounds 2012, 525, 28.

[29] R.P. Mahajan, K.K. Patankar, M.B. Kothale, S.C. Chaudhari, V.L. Mathe, S.A. Patil, Pramana J. Phys. 2002, 58,1115 .

[30] P.K. Sharma, R.K. Dutta, A.C. Pandey, Adv. Mat. Lett. 2011, 2, 246.

[31] H. Zhao, Z. Zhang, Z. Zhao, R. Yu, Y. Wan, M. Lan, Adv. Mat. Lett. 2011, 2, 172.

[32] M. Prabaharan, JF. Mano, Macromol. Biosci. 2005, 5, 965.

[33] H.K. Patra, S. Banerjee, U. Chaudhuri, P. Lahiri, A.K. Dasgupta, Nanomedicine 2007, 3, 111.

[34] L.H. Hurley, Nat Rev Cancer 2002, 2, 188

[35] H.K. Patra, A.K. Dasgupta, Nanomedicine 2012, 8, 842

[36] M. Nidhin, S.S. Nazeer, R.S. Jayasree, M.S. Kiran, S.K. Janardhanan, RSC Adv 2013, 3, 6906.

[37] U.I. Tromsdorf, N.C. Bigall, M.G. Kaul, O.T. Bruns, M.S. Nikolic, B. Mollwitz, H. Weller, Nano letters 2007, 7, 2422.

[38] N.H. Cho, T.C. Cheong, J.H. Min, J.H. Wu, S.J. Lee, D. Kim, J.S. Yang, S. Kim, Y.K. Kim, S.Y. Seong, Nature Nanotechnology, 2011, 6, 675. 


\section{ADVANCED \\ HEALTHCARE \\ YATERAS}

Submitted to

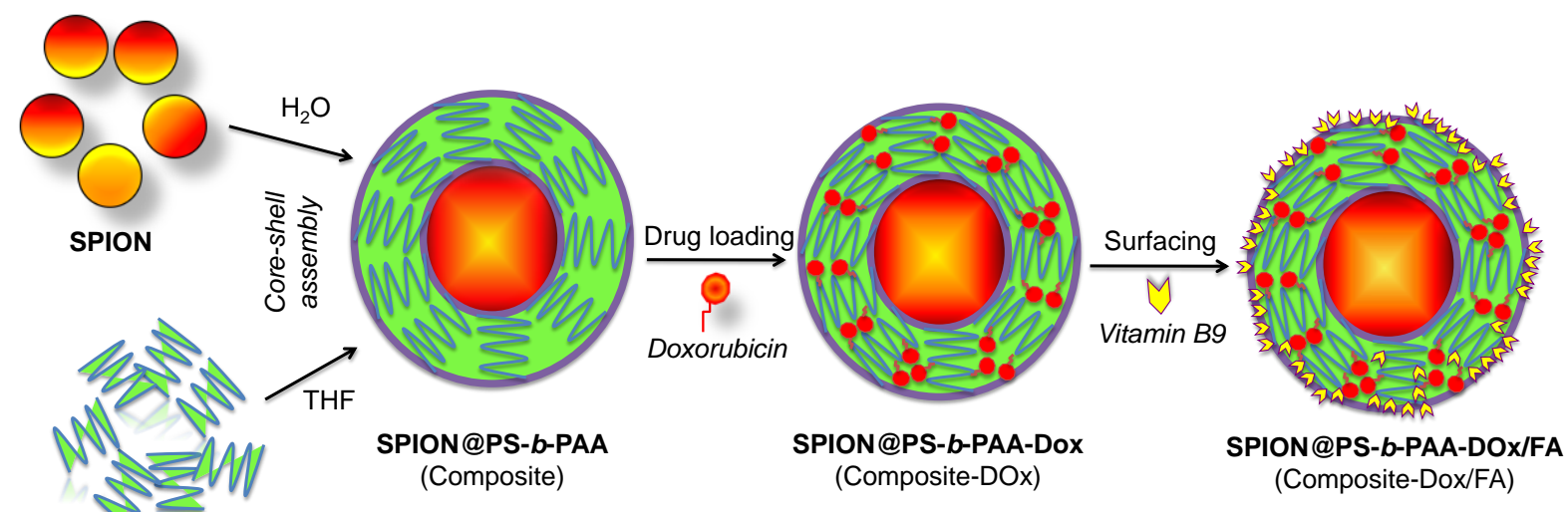

PS- $b$-PAA

(Hydrazide terminated)

Scheme 1. Fabrication of the MRI visual SPION@PS- $b$-PAA-DOx/FA cancer nano- theranostics module. 
Submitted to

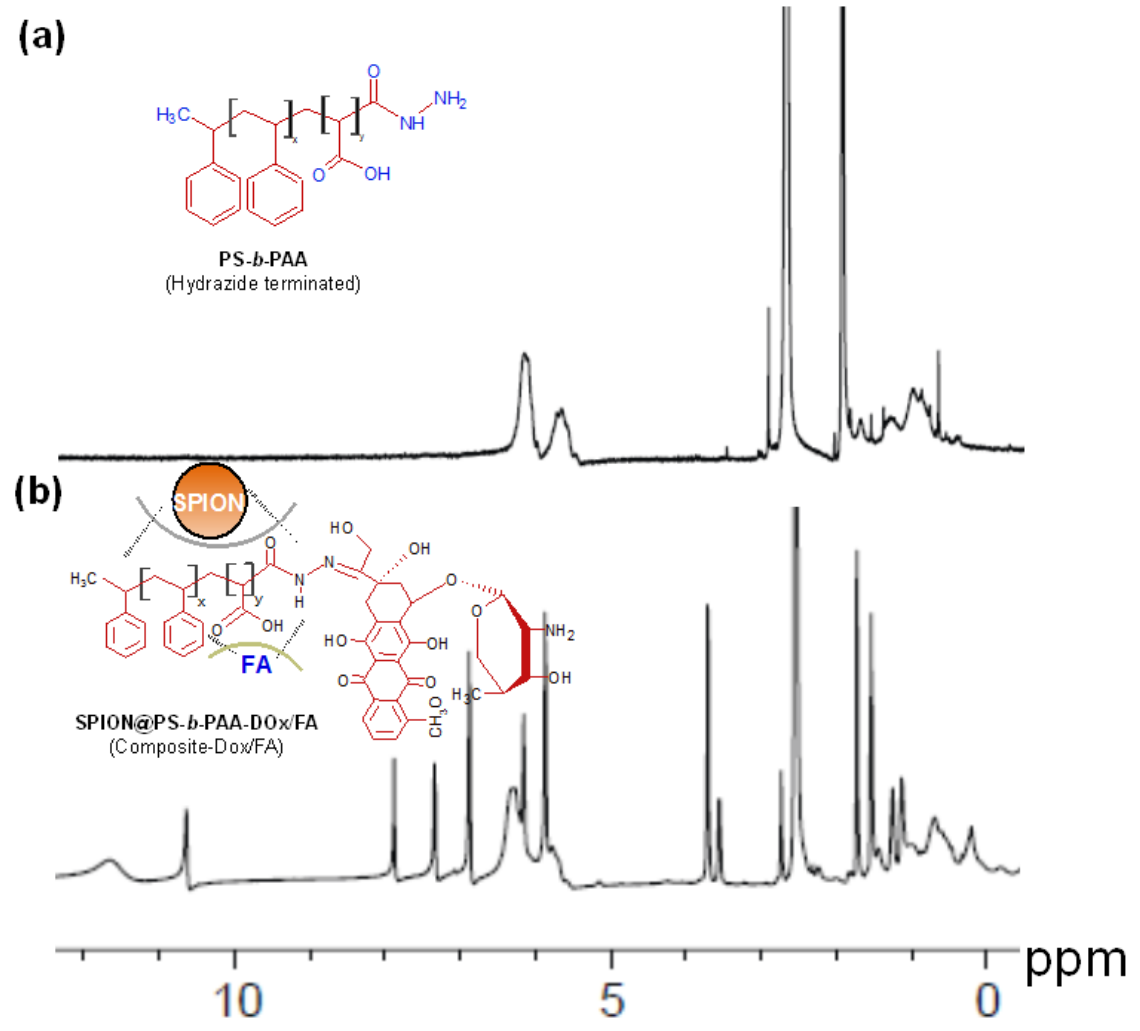

Figure 1. ${ }^{1} \mathrm{H}$ NMR spectra of (a) PS- $b$-PAA and (b) SPION@PS- $b$-PAA-DOx/FA. 


\section{ADVANCED \\ HEALTHCARE \\ Submitted to}

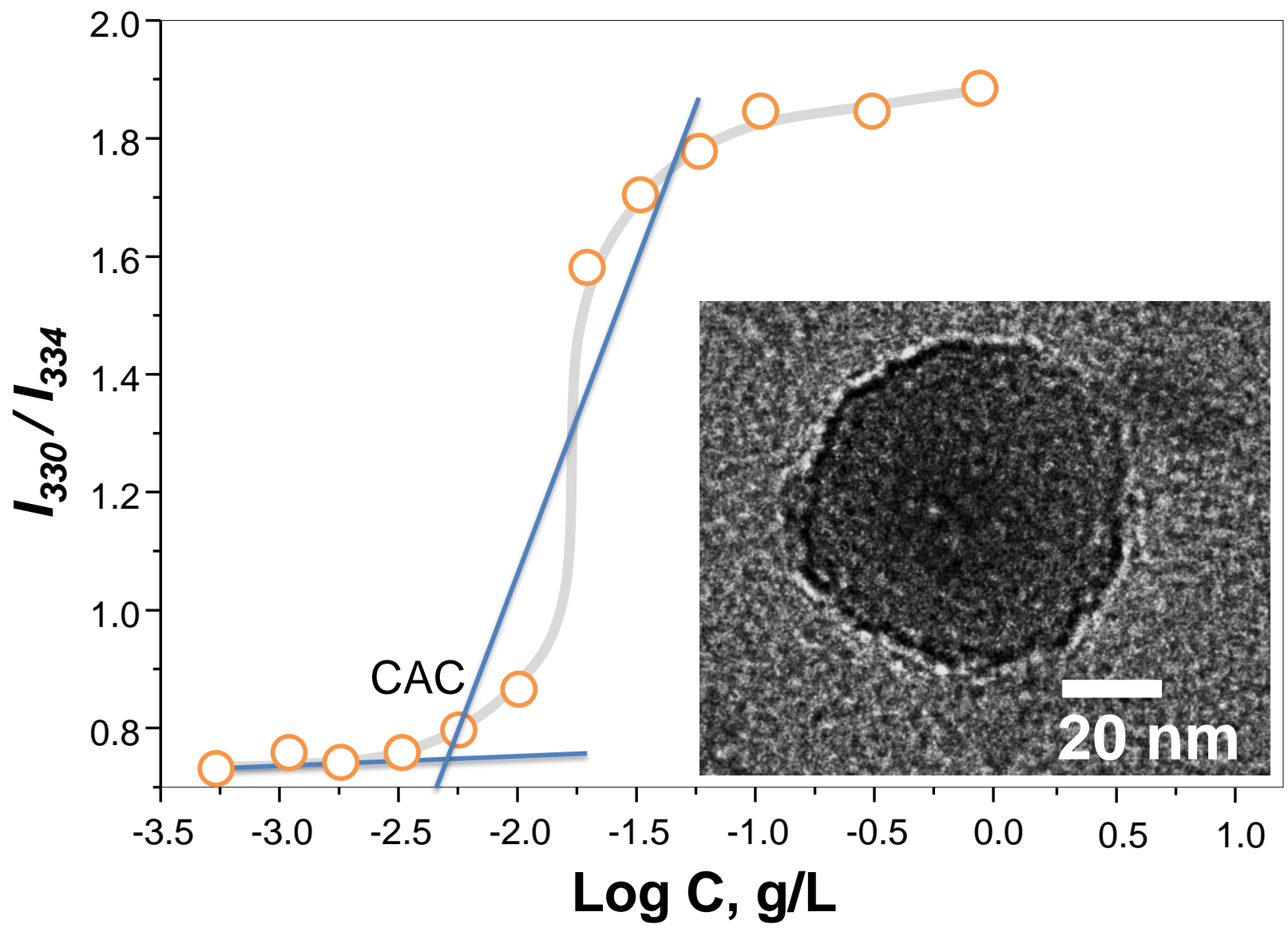

Figure 2. Plot of the intensity $I_{330}$ and $I_{334}$ ratio of the pyrene excitation spectra vs. logarithm of SPION@PS- $b$ PAA-DOx/FA concentration. Inset at at right hand side TEM image of the micelle SPION@PS- $b$-SPION@PS- $b$ PAA-DOx/FA. 

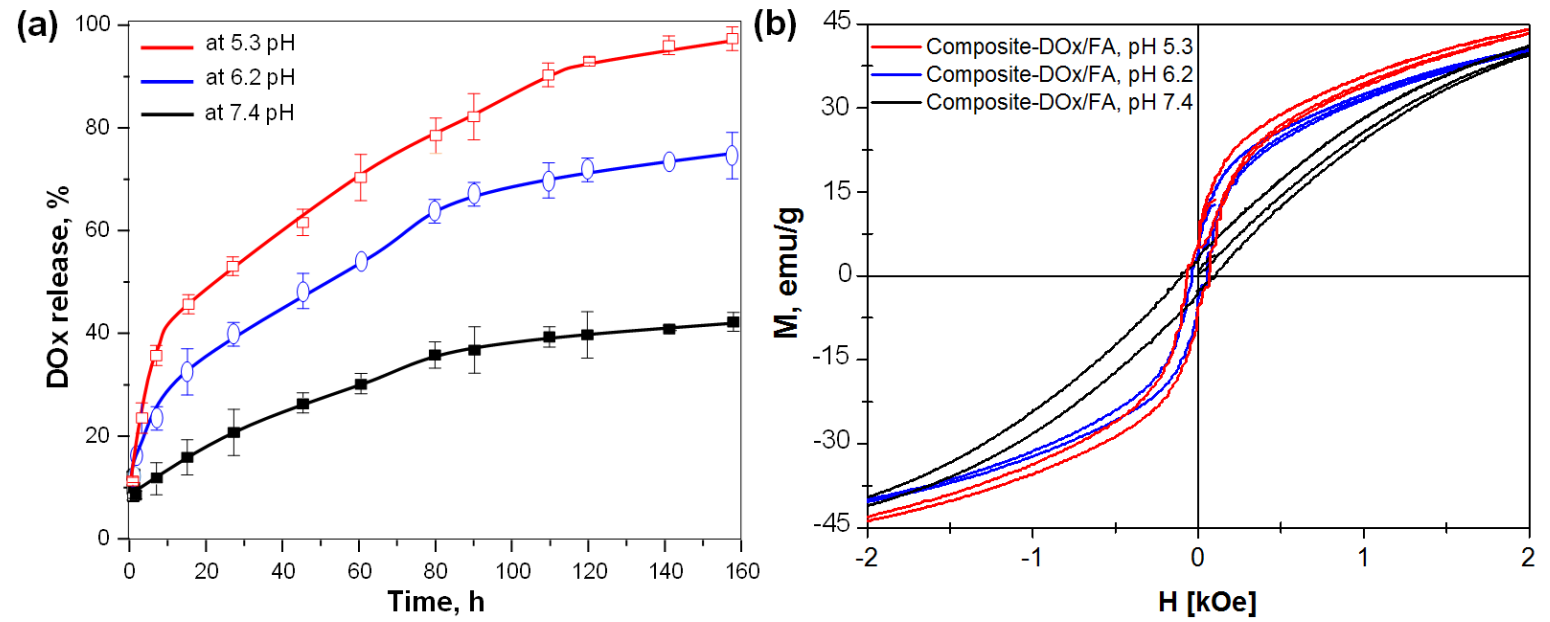

(c)
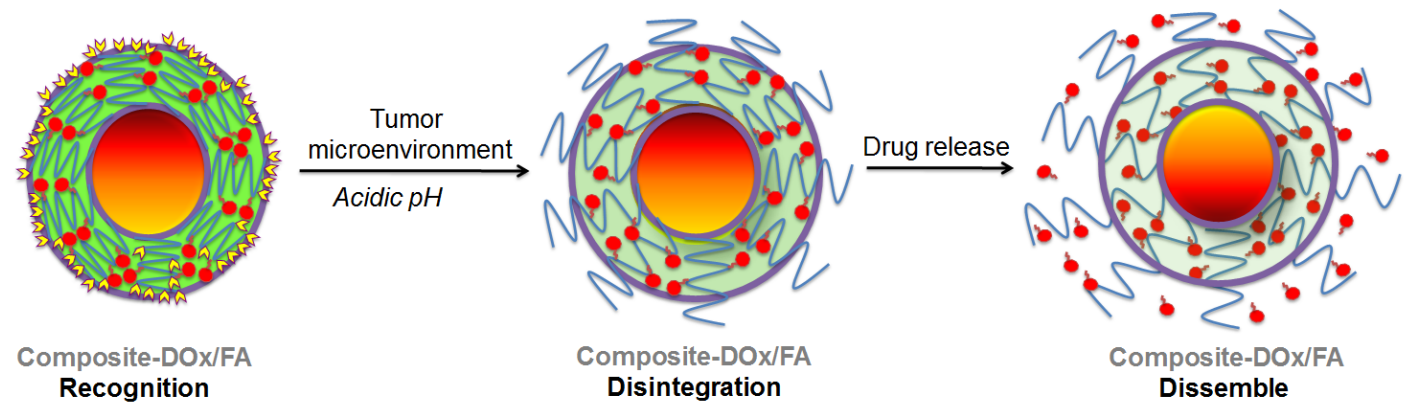

Figure 3. (a) In vitro drug release profile of DOx from the SPION@PS-b-PAA-DOx/FA core shell micelles at $\mathrm{pH}$ 5.3, 6.2 and 7.4 at $37^{\circ} \mathrm{C}$ in PBS, (b) Effect of pH on the magnetisation of SPION@PS-b-PAA-DOx/FA core shell micelles at $37^{\circ} \mathrm{C}$ and (c) The drug release mechanism via functional outcome of the $\mathrm{pH}$ response illustrated in the schematic diagram. 
Submitted to $A$ A

(a)
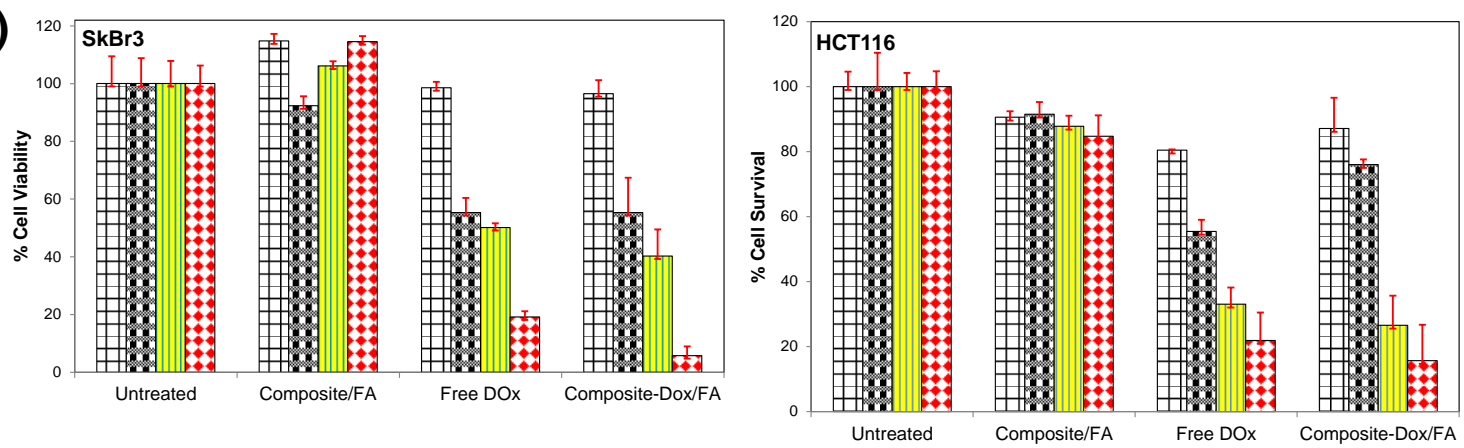

(b)
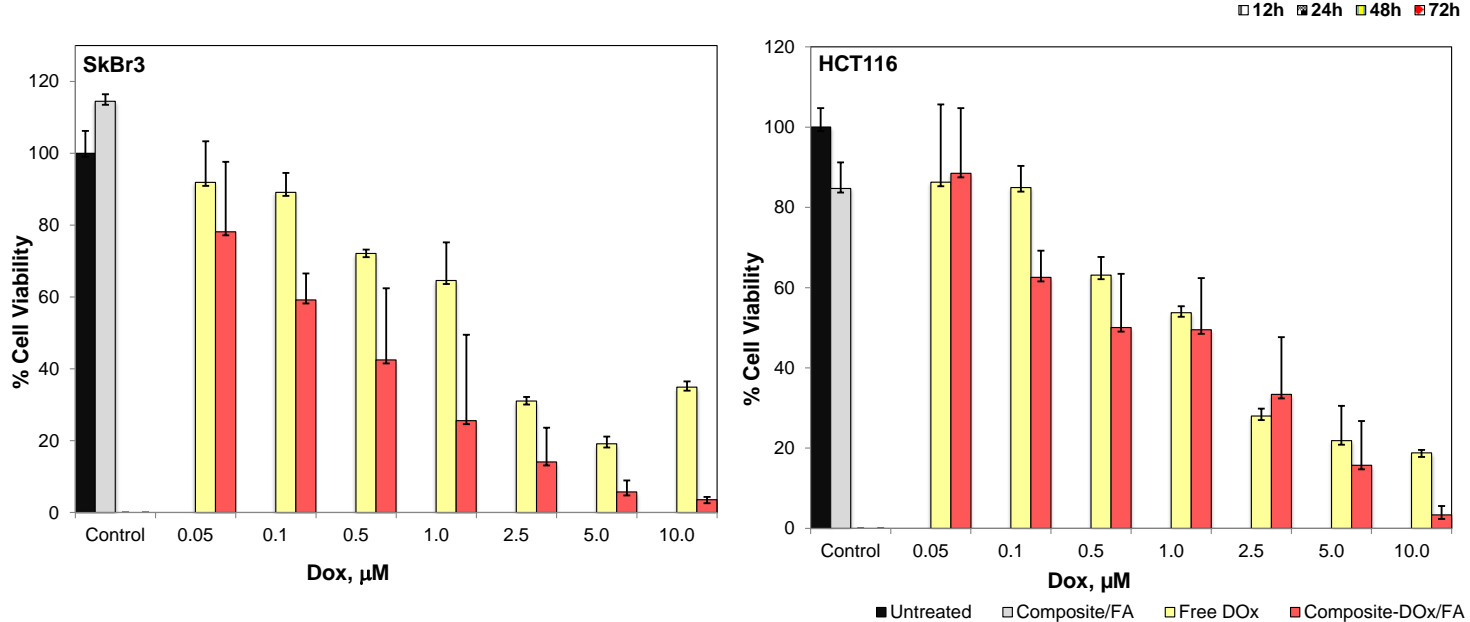

Figure 4. (a) Time and (b) concentration dependent cytotoxicity profile of composite/FA (SPION@PS-bPAA/FA), free DOx and composite-DOx/FA (SPION@PS-b-PAA-DOx/FA. The time-dependent cell culture shows comparative response to the drug at $5 \mu \mathrm{M}$ DOx dose; and the concentration dependent response was observed at $72 \mathrm{~h}$. 


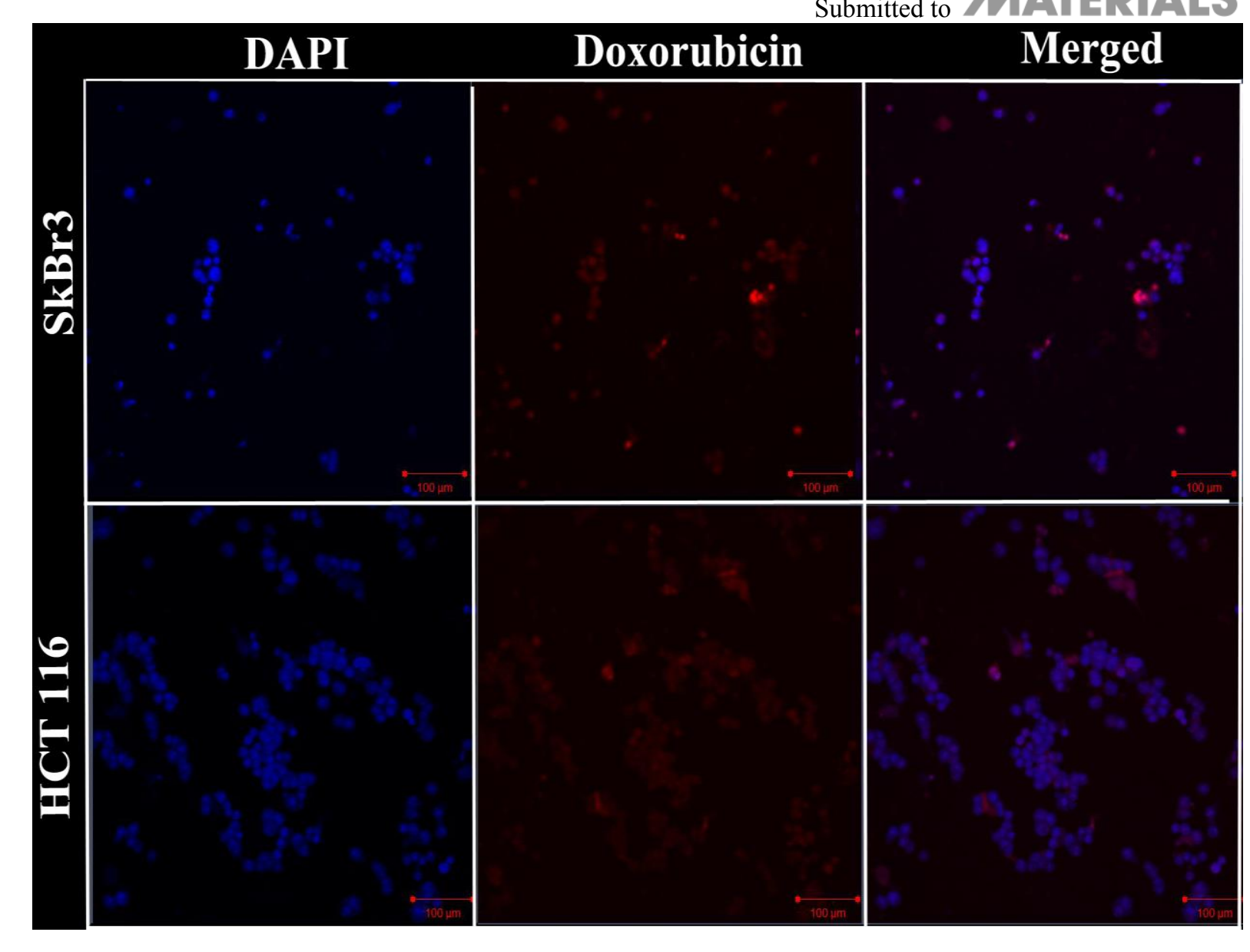

Figure 5. Micrographs of the uptake and drug localisation using confocal microscopy. Visualisation of DOx uptake (red) into to the nucleus (blue) was performed. The selected area overlap coefficient was evaluated using Zen 2009 confocal imaging software by the pixel intensity diagram of red (DOx) and blue (nucleus) pixel plan. The overlap coefficient for SKBR3 and HCT 116 cell lines treated with the composite drug were found to be 0.75 and 0.89 


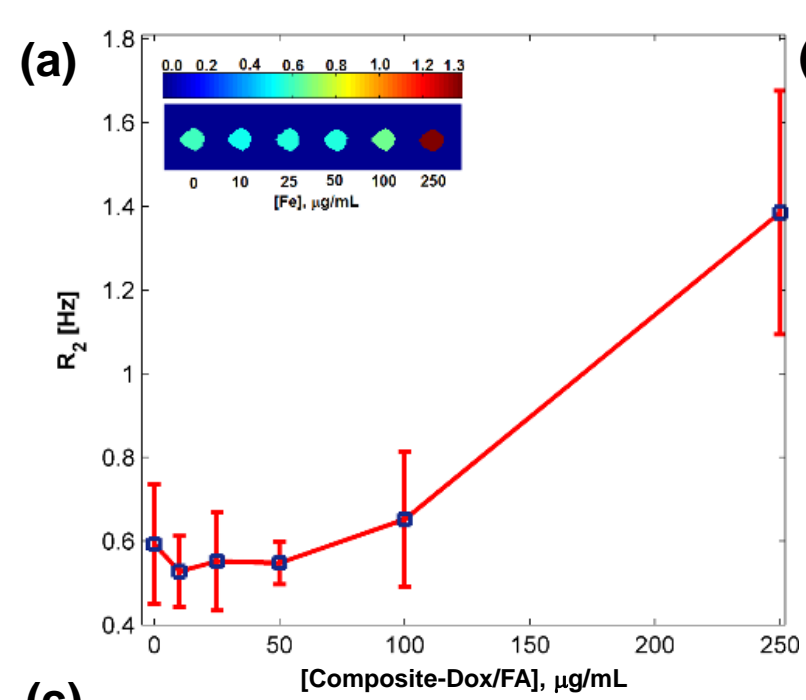

Submitted to

HEALTHCARE
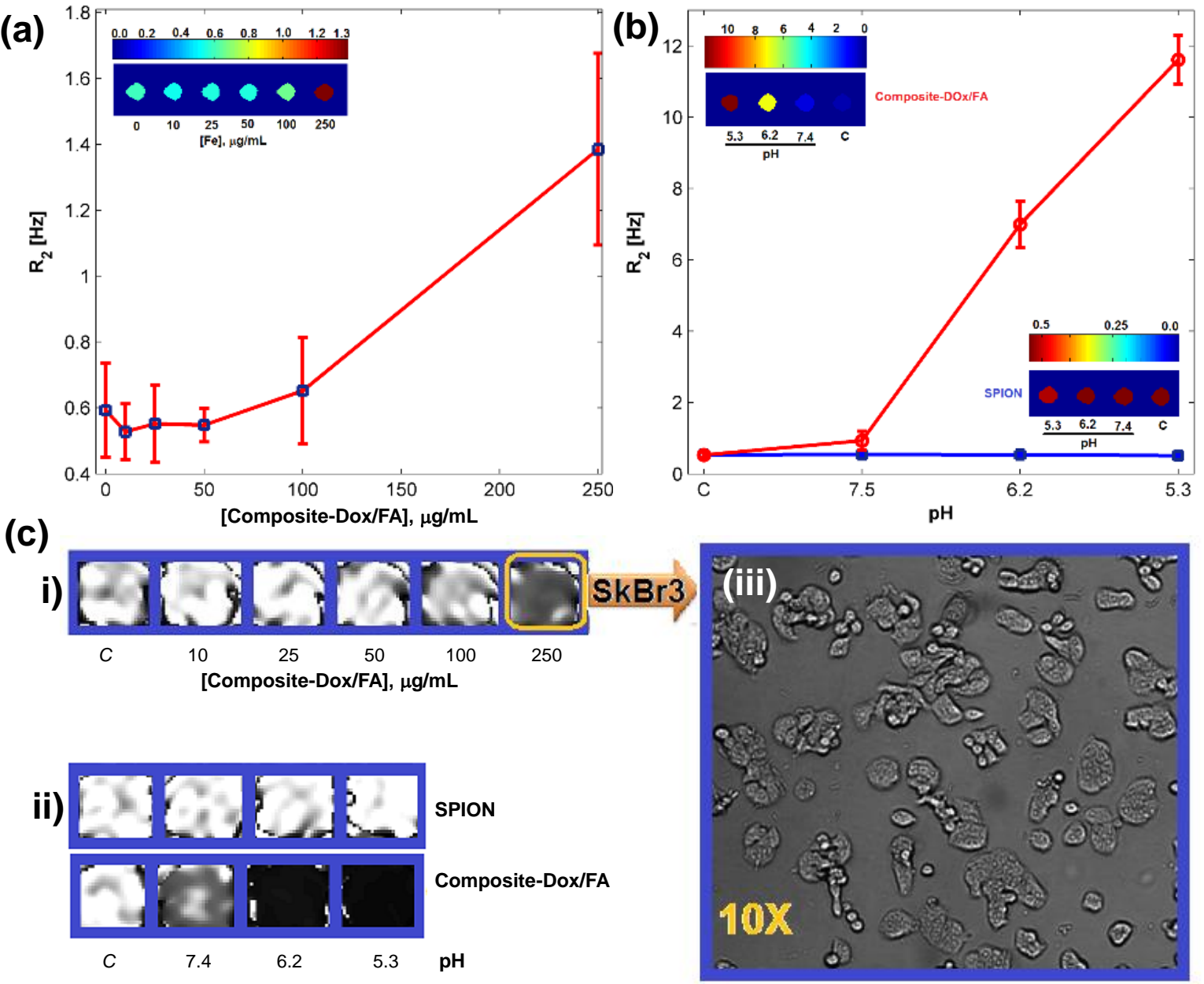

Figure 6. The MR contrast images for all the experiments were measured at room temperature having Repetition time $(\mathrm{TR})=5000 \mathrm{~ms}$; echo time $(\mathrm{TE})=20$ to $200 \mathrm{~ms}$ with an interval of $20 \mathrm{~ms}$ in a magnetic field of $1.5 \mathrm{~T}$. The inset coloured phantom images of the well $(\mathrm{a}-\mathrm{b})$ is describing the scaled image data to the full range of the colourmap described in the upper panel and the image in lower panel. (a) The relaxation rates $\left(\mathrm{R}_{2}, \mathrm{~s}^{-1}\right)$ of the SPION internalized by the monolayer whole cell SkBr3 for 3 hours as a function of concentration (c) The relative $\mathrm{R}_{2}$ of the composite (red) and SPION (blue) with respect to PBS $(C)$ as a function of $\mathrm{pH}$ (b) In vitro $\mathrm{T}_{2}$ map of MRI images on a real time basis for composite internalized monolayer $\mathrm{SkBr} 3$ cells i). The relative $\mathrm{T}_{2}$ map of the $\mathrm{pH}$ dependent contrasting ability of the composite (lower panel) with respect to SPION (upper panel) in ii). The respective phase contrast image of the $\mathrm{SkBr} 3$ cluster is shown in iii). 
 \\ Submitted to}

\section{The table of contents entry}

A novel MRI visual order-disorder nanostructures for cancer nanomedicine explores $\mathrm{pH}$-trigger mechanism for theragnosis of tumour hallmark functions. The $\mathrm{pH}$ tunable order-disorder transition induces the relative changes in MRI contrast. The outcomes elucidate the potential of this material for smart cancer theranostics by delivering non-invasive real-time diagnosis, targeted therapy and monitoring the course and response of the action.

Keywords: Custom-fit nanomedicine, Tumour microenvironment, Smart nanotheranostic; Personalised therapeutics.

Title: MRI-visual order-disorder micellar nanostructures for smart cancer theranostics

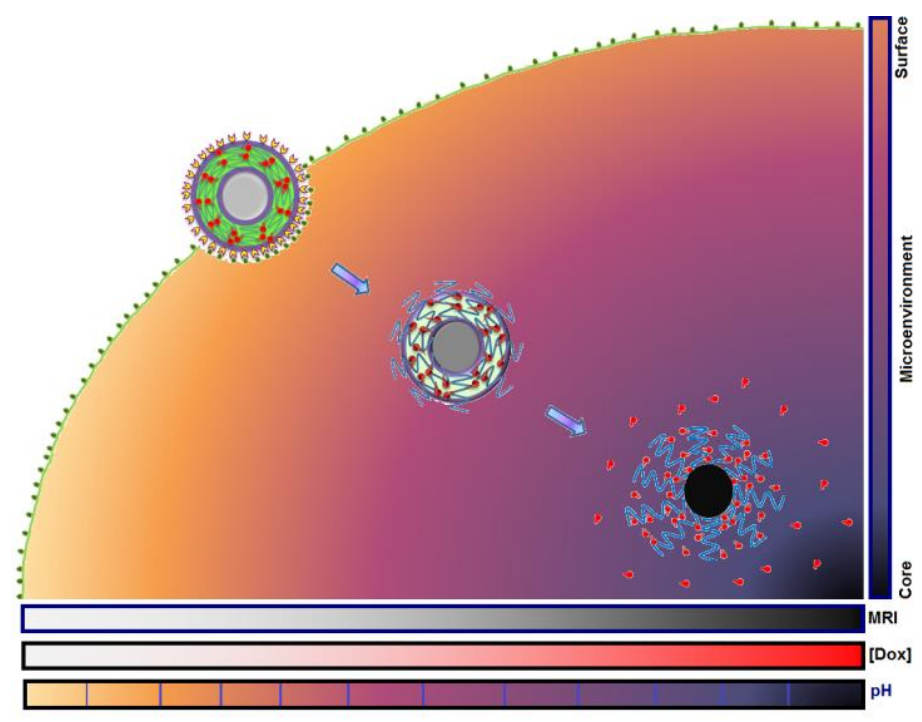




\section{Supplementary information}

\section{Matlab script for MRI:}

\section{Script1: Segmentation of area of interest}

o Load data

load ('SPION2.mat')

\% Find a good threshold separating the samples from the background. This is performed by using the Otsu's method on the maximum value from each echo. th $=$ graythresh $\left(T E \_a l l(:) . / \max \left(T E \_a l l(:)\right)\right) .{ }^{*} \max \left(T E \_a l l(:)\right)$;

\% Segment the samples from the background

mask $=$ TE_all $(:,:, 1)>$ th; $\frac{\circ}{0}$ Threshold the first image

mask = imdilate (mask, ones(3)); \% Grow mask one pixel

mask = imfill (mask,'holes'); Fill holes

mask = imerode (mask, ones(3)); $\frac{\circ}{0}$ Remove borders for better estimate

mask = imerode (mask, ones(3)); $\frac{\circ}{\circ}$ Remove borders for better estimate

을 Find connected components, i.e. the regions of interest in the images $\mathrm{CC}=$ bwconncomp (mask);

o Save the mask and the struct with region data

save SPIONmask.mat CC mask 
Script2: Calculation of $\mathrm{R}_{2}$

\section{Submitted to}

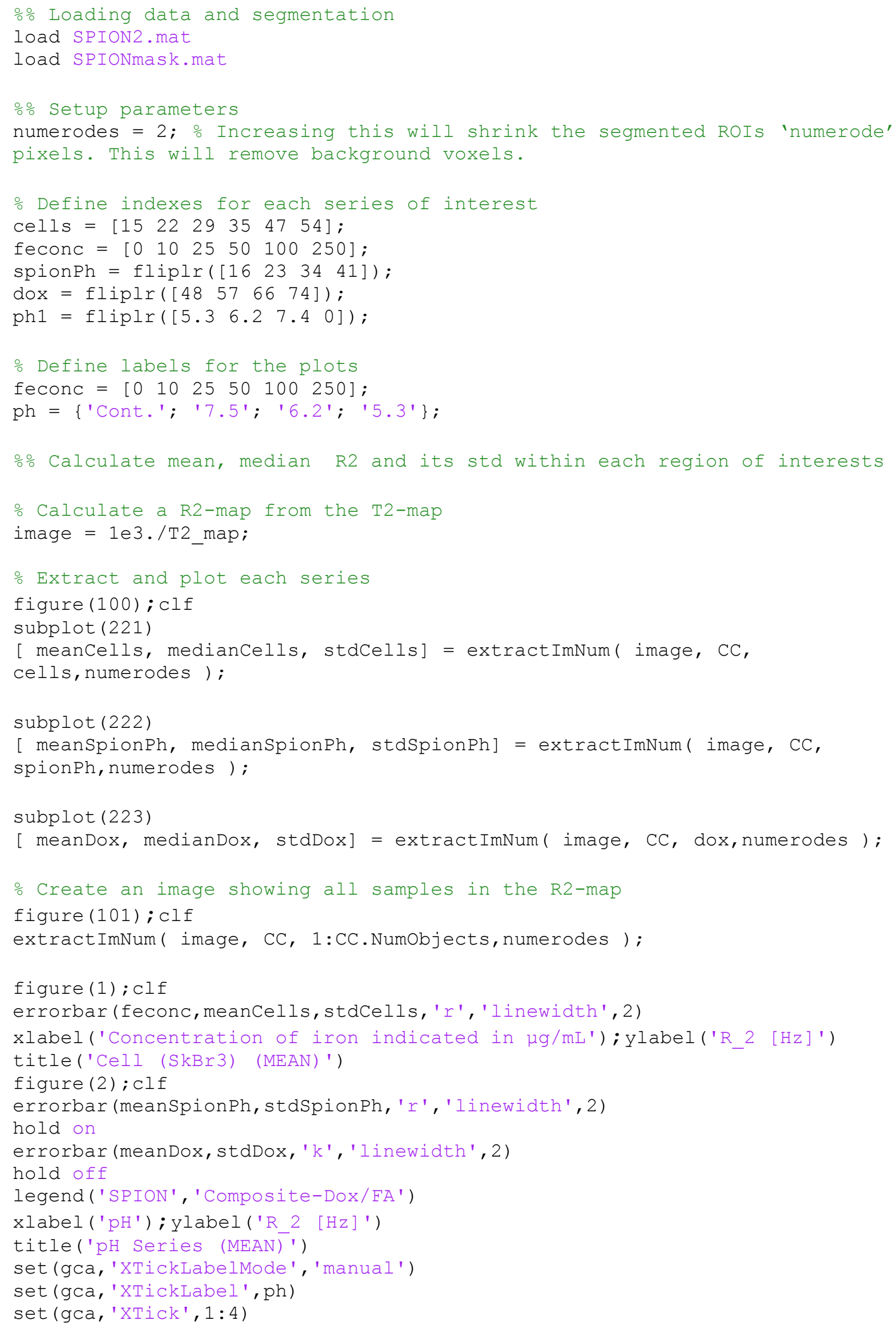




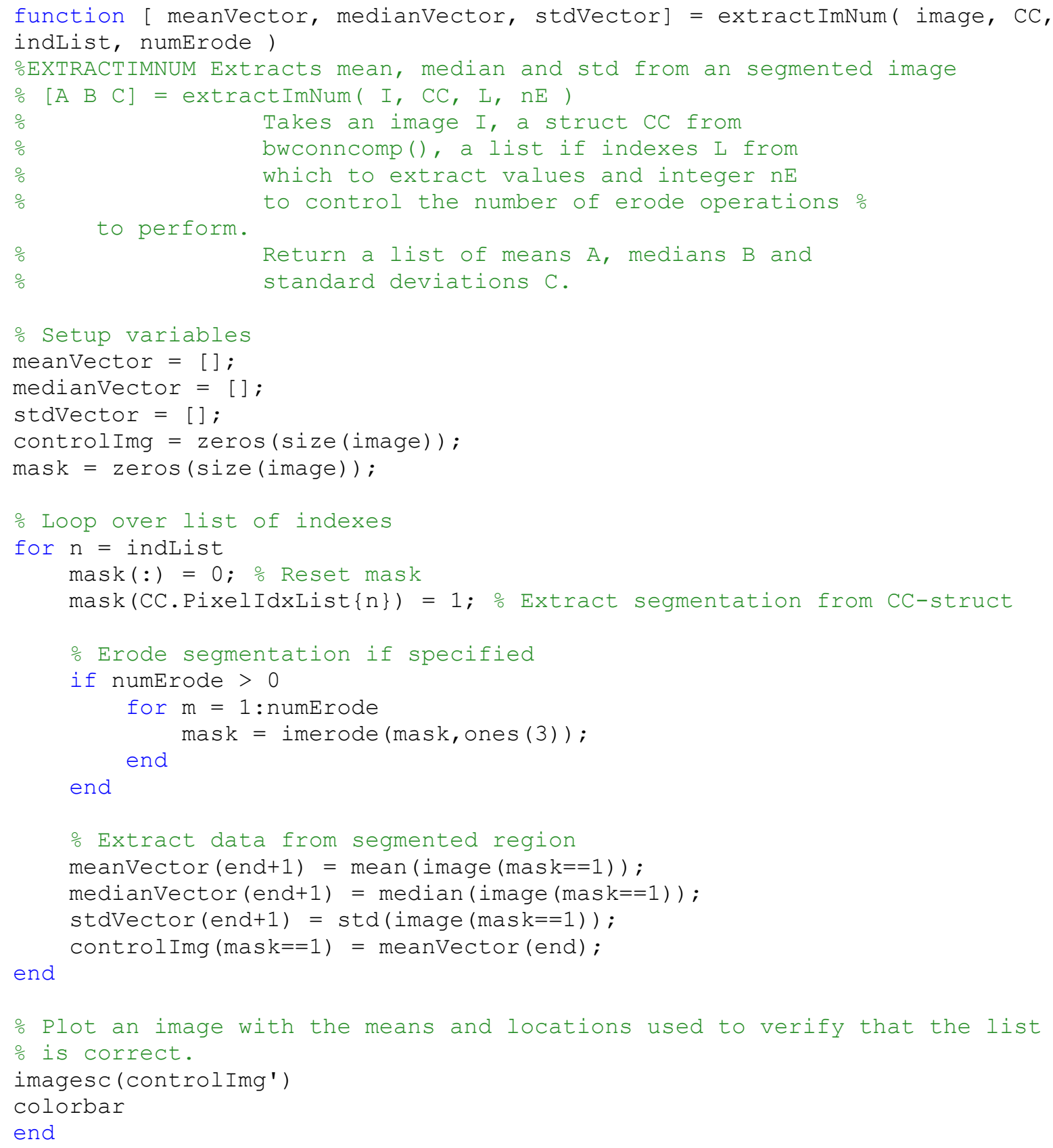

